$\begin{array}{ll}\text { le portiQue } & \text { Le Portique } \\ \text { Revue de philosophie et de sciences humaines } \\ \text { e-Portique | } 2005\end{array}$

\title{
Pavillon cuivré aux ambiguïtés martiales
}

\section{Francis Rousseaux}

\section{OpenEdition \\ Journals}

Édition électronique

URL : https://journals.openedition.org/leportique/523

DOI : 10.4000/leportique.523

ISSN : $1777-5280$

\section{Éditeur}

Association "Les Amis du Portique"

\section{Référence électronique}

Francis Rousseaux, «Pavillon cuivré aux ambiguïtés martiales », Le Portique [En ligne], e-Portique, mis en ligne le 09 juin 2005, consulté le 13 septembre 2022. URL : http://journals.openedition.org/ leportique/523 ; DOI : https://doi.org/10.4000/leportique.523

Ce document a été généré automatiquement le 13 septembre 2022.

Tous droits réservés 


\title{
Pavillon cuivré aux ambiguïtés martiales
}

\author{
Francis Rousseaux
}

À mon compagnon de voyage Bémadji Koulro-

Bezo

1 Cet article fait pendant à l'article intitulé «À l'embouchure jubilatoire de la trompette ", téléchargeable à l'adresse http://recherche.ircam.fr/equipes/sel/ rousseaux/autres $2 . \mathrm{htm}$

2 Je relis ces pages dans le Thalys qui me conduit vivement à Bruxelles pour une journée de travail dans les locaux de la Commission européenne. Le TGV est peuplé de cadres préoccupés qui vont à leurs affaires. Seul mon voisin de banquette s'accorde le temps de jeter un œil aux plaines du Nord à peine rompues par quelques terrils. Il se lève et revient bientôt avec un café qu'il entreprend de siroter bruyamment, et un sandwich qu'il déballe amplement sur sa tablette.

3 Pourquoi les moindres écarts de nos voisins immédiats sont-ils à ce point agaçants et saugrenus, voire franchement irritants, alors que dès lors qu'on part à l'aventure, les mille détails exotiques qu'on remarque sont amusants et nous attachent avec sympathie aux personnages qui les déploient ? Pourquoi suis-je mal disposé envers mon voisin de Thalys qui boit son café un peu bruyamment alors que je sympathise d'emblée avec le marchand de chèvres de l'occasion de marché ${ }^{1}$ N'Djamena-Bomboyo qui m'impose d'autorité deux chevreaux sur le plastron?

Pourquoi m'intéressai-je à la condition de vie des Tchadiens, alors que ma propre vie est ailleurs ? Sur quoi repose ultimement cette étrange disposition?

Le but de cet article est de mettre en perspective ce dernier point.

$I^{\circ}$ Rien sur ma trompette

6 En route pour l'Aéroport Roissy-Charles-de-Gaulle en cette fin d'année 2004, que savaisje du Tchad ? J'avais certes lu le célèbre Le retour du Tchad de Gide, écouté sur FranceInter quelques jours auparavant l'excellente émission Rendez-vous avec $X$ consacrée à l'histoire contemporaine de ce pays ... Mais dans mon bagage j'apportais, coincés entre 
une pharmacie portative et une panoplie de broussard aguerri, Le cru et le su de Jean Pouillon, Logique de la sensation consacré par Deleuze à la peinture de Francis Bacon, ainsi qu'un traité d'improvisation-jazz pour le piano: à croire que je renonçais à préparer ce voyage, m'en remettant à ma bonne étoile pour naviguer au mieux dans cette région du Sahel si peu visitée, seulement réputée pour être l'une des plus pauvres du monde.

Viatique pour N'Djamena

7 Comme tout un chacun, j'avais lu quelques récits de chasse/massacre aux grands fauves de l'époque coloniale, et je me souvenais vaguement de quelques épisodes épiques de la constitution par le futur maréchal Leclerc des forces armées africaines qui allaient plus tard libérer Paris de l'occupation allemande. Mais pour le reste, se bousculaient pêlemêle dans mon imaginaire :

8 Certains événements marquants de la colonisation de l'Afrique Équatoriale Française et des influences néo-coloniales françaises dans la région après 1960 (la présence de l'armée française non loin de N'Djamena via la fameuse base aérienne « Épervier »);

9 Les tensions Nord-Sud entre les nomades-éleveurs islamisés et les sédentairesagriculteurs chrétiens et/ou animistes, typiques du Sahel (l'enlèvement des époux Claustre en 1974 et leur longue détention par les Toubous emmenés par Hissène Habré dans le Tibesti);

10 La fuite d'Hissène Habré au Sénégal et son remplacement par Idriss Déby en 1991, à la tête d'un État sans nation (la découverte de gisements de pétrole dans le Sud du pays et le succès américains d'Exon/Esso à s'en emparer intégralement, au détriment du prétendant français Elf).

11 Il est vrai aussi (et ma curiosité pour ce pays n'y était sans doute pas étrangère), que j'avais développé par le passé une collaboration professionnelle avec un Attaché militaire français qui avait été en poste à l'Ambassade de France à N'Djamena pour le compte de la Direction du Renseignement Militaire au ministère de la défense français : à cette époque, j'étais à la recherche de scénarios crédibles pour exemplifier mes théories sur les Systèmes d'Information et de Communication et leur mise en œuvre dans des applications de décision en situation de crise $^{2}$. Ce colonel en retraite m'avait fourni de précieux renseignements sur la situation géopolitique du Tchad, à partir d'événements qu'il avait lui-même parfois vécus « sur le terrain ».

12 C'est ainsi que les positions géostratégiques de Biltine, Abéché, Sarh ou Faya-Largeau, du Darfour, du lac Tchad, du Tibesti, de l'Ennedi ou de l'Ouaddaï ne m'étaient pas inconnues, tout comme les rapports de voisinage tumultueux avec la Libye, la République centrafricaine, le Nigeria, le Niger et le Cameroun. 


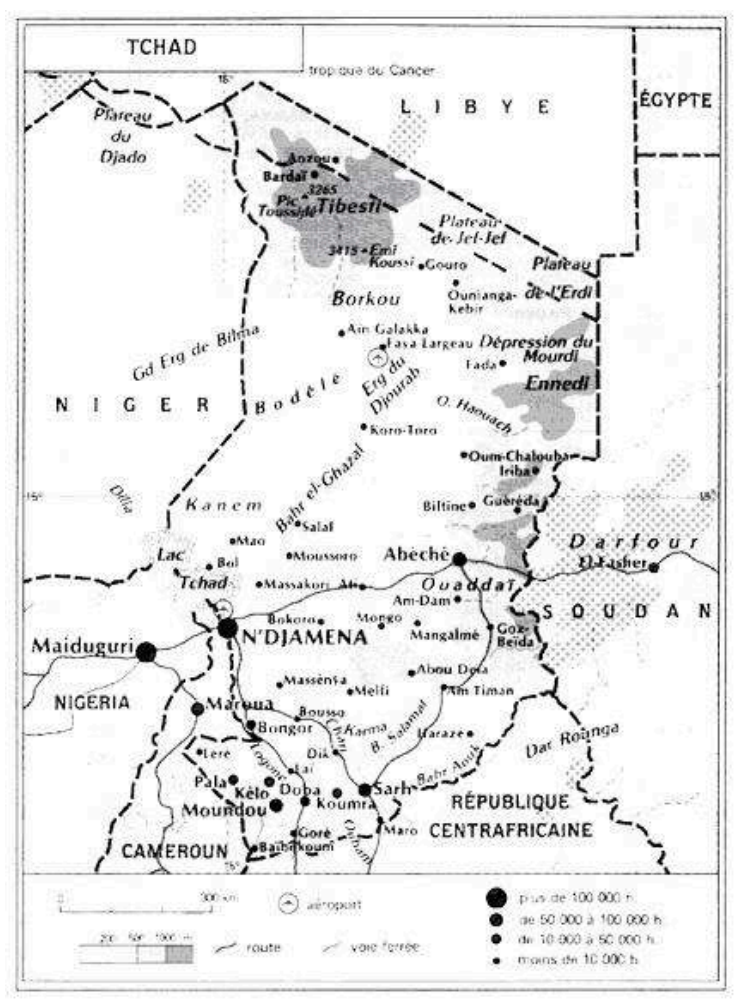

Mais tout cela était bien théorique, je n'avais jamais mis les pieds au Tchad (même si je connaissais assez bien l'Afrique du Nord et l'Afrique noire) et j'ignorais tout de la configuration humaine précise de cette région d'Afrique sahélienne : des Gorans du Nord et des Zaghawas de la région de Biltine et d'Iriba, des Saras et des Massas du Centre Ouest, des Toupouris de Léré, sans parler des Peuhls conduisant leurs troupeaux de bêtes à cornes, j'ignorais l'extraordinaire diversité.

Pour l'heure, mon passeport était bardé des visas pour le Tchad et le Cameroun, obtenus à force de patience et au prix de nombreuses visites aux ambassades parisiennes de ces pays (les quelques universitaires, baroudeurs et militaires que j'avais contactés me recommandaient tous de fuir le Tchad au plus vite pour gagner le Nord Cameroun et la région de Maroua, connue pour être plus accueillante et moins difficile à parcourir que le Tchad, les coupeurs de route étant plus rarement meurtriers, les pistes étant parfois goudronnées et la situation sanitaire du pays étant moins alarmante).

5 Et surtout j'allais retrouver un médecin français ${ }^{3}$ spécialiste de médecine tropicale, en poste pour la Coopération française dans la deuxième ville du pays, la sudiste Moundou (que je saurais bien gagner depuis la capitale, en suivant les vallées du chari et du Logone).

Premiers contacts avec le Tchad

Lorsque j'approchais le périmètre emmuré de la concession de la Coopération française dans la ville de Moundou, je séjournais au Tchad depuis plus d'une semaine, et je me sentais déjà bien acclimaté. J'avais visité N’Djamena à mon arrivée, m'étant acquitté des formalités administratives et policières avec le sang-froid qui convient. Je me levais de fort bonne heure pour admirer la lumière matinale et affronter parfois les longues heures de voyages qui sont le lot des déplacements en Afrique et particulièrement au Tchad (très peu de routes goudronnées, des pistes et des véhicules en mauvais état, des 
chargements rocambolesques et une organisation folklorique, quoique finalement convenable compte tenu de la teneur et de la variété des difficultés bravées).

Je m'arrangeais pour faire la sieste et éviter les grosses chaleurs ${ }^{4}$, me couchais tôt pour prendre du repos et éviter les trajets nocturnes (il n'y a pratiquement pas d'électricité au Tchad, les quelques groupes électrogènes abandonnés à l'indépendance par les Français peinant à fournir le pays même par intermittence, et l'électricité y est l'une des plus chère du monde, hors de portée des bourses). Je savais remplir ma gourde et fabriquer de l'eau potable en y adjoignant des pilules de produit chloré, je savais choisir ma nourriture de façon à éviter les risques d'intoxication graves et laissais derrière moi la première vague de problèmes intestinaux.

J'avais été tout de suite émerveillé par la beauté et la variété des populations, leur gentillesse et leur dignité aussi, et bien entendu j'étais passionné par des scènes africaines du marché, du portage des denrées, émerveillé aussi par les couleurs des pagnes et autres boubous, les sourires éclatants des jeunes gens. J'étais un nasara ${ }^{5}$, un patron, un papa, un blanc heureux de découvrir ce pays, ou tout au moins une partie de sa zone sahélienne et semi-désertique, les montagnes du Nord étant pour longtemps inaccessibles ${ }^{6}$.

19 Je venais de franchir quelques cinq cents kilomètres de brousse désertique, compressé parmi dix-neuf personnes dans des minibus à neuf places, sans compter les chèvres et les poules. J'avais fait escale à Bangor, en face de la ville camerounaise de Yagoua, et visité à plusieurs reprises les bords des fleuves Chari et Logone, splendides fleuves africains, nonchalants en cette saison, au bord desquels les gens se baignent et lavent leur linge, naviguent à l'aide de pirogues creusées dans des troncs. Plus que par les cultures maraîchères sommaires pratiquées au bord du fleuve, j'étais très intéressé par l'activité millénaire de confection des briques en argile crues ou cuites ${ }^{7}$ servant à la construction des cases, et pratiquée tout au long des fleuves, souvent même par des enfants, peu scolarisés dans la région.

De même, j'avais suffisamment discuté avec des autochtones et des coopérants civils de plusieurs nationalités, suffisamment observé de scènes de la vie quotidienne pour me faire une première idée sur la situation politique du pays. En gros, les Zaghawas ${ }^{8}$ d'Idriss Déby, héritiers de la tradition sahélienne du Nord, nomades éleveurs de chameaux représentant quelques pour cent de la population du pays, de religion islamique, détiennent le pouvoir et le font régner sans partage, notamment à l'encontre de leurs rivaux traditionnels du Sud. C'est ainsi que tous les cadres de l'état sont Zaghawas, et que règne une corruption galopante dans tous les secteurs de l'économie. Le fait que le sudiste Tombalbaye, premier président du Tchad indépendant, ait régné en son temps de manière tout aussi partiale, ne change rien à l'affaire.

21 C'est ainsi notamment que le Sud du pays, plus riche et autrement organisé, est empêché de se développer. L'exploitation des champs de pétrole sudistes, par exemple, ne donne lieu à aucun emploi et à aucune richesse directe pour les Tchadiens du cru : le pétrole est acheminé à l'état brut à travers un oléoduc enfoui qui traverse le Cameroun jusqu'au terminal off-shore de Kribi (plus de deux mille kilomètres de longueur), avant d'être chargé dans les pétroliers américains. Les dividendes, loin de s'en retourner aux Tchadiens (qui vivent dans des conditions de pauvreté effarantes, leur espérance de vie étant moitié moindre que celle des habitants des pays riches), vont plutôt à leurs oppresseurs Zaghawas, qui parviennent à contourner les garde-fous mis en place par le 
Fonds Monétaire International pour acheter des armes et les utiliser pour réprimer les opposants.

De même, une grande part de l'aide civile au développement en provenance des organisations internationales est détourné ou spolié. Seules peut-être quelques Organisations Non Gouvernementales (ONG) de dimension internationale ${ }^{9}$ et des petites organisations de proximités comme les missions évangéliques, agissant au long cours, peuvent résister à la pression. Les dispositifs de coopération liés aux états étrangers sont mal placés, comme le cas des services de coopération française ${ }^{10}$ l'illustrera bientôt de façon tragique.

Bienvenu à l'hôpital de Moundou

J'étais très heureux de retrouver Pierre, qui loge dans une petite maison de la concession de la coopération française à Moundou, la deuxième ville du pays. Rien de luxueux ici, mais des murs en dur, un groupe électrogène, quelques arbres mal entretenus, qui contrastent pourtant avec les pistes en latérite de la ville encombrée de détritus en tout genre et parcourus par les chèvres, moutons, bœufs, ânes, porcs et poules en liberté surveillée. Alentour, une population avenante ${ }^{11}$, à l'exception des quelques buveurs de bili-bili (la bière de mil "maison" et servie dans de vastes ramequins) qui certains soirs peuvent boire jusqu'à perdre provisoirement leur sens de l'hospitalité.

Passées les civilités du premier jour, je compris peu à peu, à fréquenter les rares coopérants français expérimentés habitant la concession, que la situation qu'ils vivaient au Tchad était une des plus difficiles qu'ils avaient jamais rencontrées. Pierre, qui avait travaillé plusieurs années en Afghanistan pour le compte de MSF, dans les pires moments de la guerre contre les Soviétiques, parcourant le pays à cheval pour y soigner des réfugiés, prétendait que la situation actuelle au Tchad était plus terrible encore.

Il faut dire que ce médecin travaillait pour la première fois pour le compte du gouvernement français au titre de la coopération. En réalité, cela faisait plusieurs mois qu'il ne quittait pratiquement plus sa maison, et qu'il ne se rendait à son bureau voisin que quelques fois par semaines pour y saluer cordialement quelque employé désœuvré. Il évitait également de se rendre aux rares réunions de travail dans la capitale. Il faut dire aussi que son dernier voyage avait été mouvementé : il avait alors essuyé un tir d'arme automatique qui avait blessé son passager et pulvérisé le pare-brise de son véhicule tout terrain. Il faut dire encore que Pierre n'était pas certain de pouvoir écarter l'hypothèse d'avoir été pris pour cible pour des raisons liées à ses prises de position professionnelles, et non pour de banales raisons de banditisme ${ }^{12}$.

En effet, Pierre était en froid avec sa hiérarchie française et, partant, avec les dignitaires tchadien dont il était officiellement le conseiller.

Dès son arrivée dix-huit mois plus tôt, en sa qualité de Conseiller technique du Délégué gouvernemental santé (tchadien) de la région du Logone occidental (huit pour cent de la population du pays soit environ six cent mille habitants), il avait diagnostiqué une épidémie de choléra que les autorités n'avaient pas voulu reconnaitre, et il avait du juguler seul l'épidémie, avec la désapprobation des autorités sanitaires du pays. Par la suite, il avait mis à jour une terrible corruption pratiquée à grande échelle à l'hôpital de Moundou, le deuxième hôpital du pays. Dans cet hôpital, de nombreuses transfusions sanguines sont pratiquées ${ }^{13}$ avec du sang non-testé et donc contaminé dans quelque trente pour cent des cas $^{14}$ par le virus du SIDA. Pierre s'est aperçu de ces pratiques et a 
facilement chiffré les morts d'homme qu'elles entraînent. "La chose est horrible ", me direz-vous, « mais on peut comprendre qu'il soit acceptable, en cas de force majeure, de sauver la vie de quelqu'un au prix de sa mort à dix ans ». Soit, mais Pierre a aussi découvert que les services de la Coopération française offraient chaque année à l'hôpital les produits et équipements nécessaires pour dépister le VIH dans le sang prélevé sur les donneurs, et que ces produits étaient revendus dans les établissements privés (pharmacies ou cliniques et même échoppes de bazar) tenus par les cadres de l'hôpital.

Qu'auriez-vous fait à sa place ? Il a rédigé un rapport à ses supérieurs hiérarchiques en évoquant de façon discrète ses découvertes et en demandant des conseils sur l'attitude à adopter. On lui a répondu en substance qu'il fallait fermer les yeux, et rédiger plutôt le plan de formation des personnels tchadien ${ }^{15}$ qu'on attendait de lui sans plus tarder. Pierre s'est exécuté, mais il a tout de même envoyé un rapport circonstancié à l'Ambassade de France à N'Djamena, un certain soir où le courrier électronique fonctionnait, par le truchement d'un courriel approprié.

Le lendemain de l'expédition de son courriel, il a été convoqué par le directeur tchadien de l'hôpital de Moundou, qui l'a menacé de graves ennuis s'il continuait à s'intéresser de trop près à la gestion des dons de la coopération française à l'hôpital. Quelque temps plus tard, sa voiture était mitraillée, sans qu'on puisse affirmer que les deux événements soient liés d'une quelconque manière.

II Rien sur le pavillon cuivré

30 À Moundou, je quittais la concession de la coopération française au levé du jour pour me rendre au bord du Logone, que j'aimais longer au petit matin. Je m'asseyais pour voir le soleil monter sur les eaux, regarder traverser les vastes troupeaux de vaches maigres et cornues conduites par de jeunes Peuhls nu-pieds armés d'arcs et de flèches, admirer les jeunes filles Peuhls portant le lait des troupeaux au marché, en équilibre sur leur tête dans d'immenses vasques de bois, voir travailler les confectionneurs de briques, scruter les pirogues antédiluviennes mues par des pagaies grossièrement taillées, prendre le petit-déjeuner au milieu d'ouvriers journaliers en attente d'emploi et prenant le premier soleil, ou encore de femmes occupées à la lessive de tissus colorés.

Une embouchure dangereusement métonymique

31 Ce matin-là, je doublais l'ancienne usine de la Coton-Tchad pour atteindre l'unique pont qui franchit le Logone dans la région, étroit et vétuste, emprunté par des piétons, des cyclistes et des attelages, ainsi que par quelques rares et poussifs véhicules automobiles. Peut-être dans l'espoir d'apercevoir quelques hippopotames s'ébattant dans les eaux boueuses ...

32 Je décidais de franchir le pont puis, parvenu sur l'autre rive du fleuve, de rechercher un endroit tranquille le long de la plage pour pouvoir pratiquer mon instrument de musique. Pour être plus précis, disons que j'avais pris soin d'apporter une embouchure de trompette, grâce à laquelle j'espérais bien travailler régulièrement l'émission des sons de mon instrument, qui constitue sans conteste ${ }^{16}$ sa difficulté principale. Une embouchure de trompette tient dans la main, et se présente comme une pièce de métal dense et soigneusement usinée, l'état de surface de la pièce tournée étant très régulier, brillant et argenté. Au bord du Logone, parmi les briques cuites et les pirogues archaïque, il faut bien reconnaître que l'objet a de quoi surprendre ${ }^{17}$. Mais j'étais isolé, et seuls quelques flamants roses semblaient prêter attention à mon exercice. 
33 Après une heure d'entraînement (il devait être huit heures du matin), les lèvres marquées et la peau déjà chauffée par le soleil montant, je me décidais à quitter les lieux et m'approchais du pont. J'apercevais au loin un petit attroupement de villageois qui semblait garder l'entrée du tablier. Parvenu à leur niveau, j'appris de l'un d'entre eux qu'il était impossible de traverser le pont en raison du passage prochain du président Idriss Déby. Mon instinct ne fut pas long à m'alerter sur la situation et son caractère immédiatement moins tranquille. Je fis discrètement demi-tour sans demander mon reste, avec l'idée de m'éloigner et de demander plus tard à un gamin muni d'une pirogue de me faire passer de l'autre côté du fleuve: je n'avais pas mes papiers sur moi et j'imaginais fort bien la suite probable si j'étais découvert dans les parages, muni d'une pièce métallique usinée comme tout passeport, à un moment où le président craignait pour sa vie et où sa garde rapprochée de Zaghawas tuait pour moins que cela sans être jamais inquiétée ${ }^{18}$.

Malheureusement, ma présence avait été signalée, car j'avais été suivi par un jeune homme désœuvré en possession d'un vieil appareil photo, qui attira involontairement l'attention sur moi.

Un homme en guenilles se prétendant de la Sécurité intérieure, bientôt rejoint par d'autres, m'intima l'ordre de le suivre, ainsi qu'au jeune photographe qui m'accompagnait. Difficile de se soustraire, difficile de discerner la bonne attitude. La meilleure était encore de m'exécuter. Encadré par ces drôles de policiers, je suis remonté sans discuter jusqu'à l'entrée du pont, où j'ai été remis entre les mains d'un militaire assez désagréable, qui m'a fouillé et m'a demandé mes papiers, que je n'avais pas. Découvrant sans peine l'embouchure de la trompette qu'il examinait sous toutes les coutures et soupesait longuement, me demandant des explications sur la présence à mes côtés du jeune photographe, il me conduisit jusqu'à un poste de garde installé à quelques dizaines de mètres de là, en bordure de piste.

36 Entre temps, la foule avait grossi à l'entrée du pont, faite principalement de femmes empêchées de rejoindre le marché de la ville pour y vendre leurs produits. Certaines de ces femmes prirent courageusement position pour ma libération immédiate et invectivèrent en ce sens les militaires, que quelques coups de crosses de kalachnikov eurent tôt fait de ramener à une position moins altruiste. Puis passèrent les premiers éléments des troupes spéciales du Président: Des pick-up surélevés transportant chacun une bonne dizaine de Zaghawas portant treillis et cheich, brandissant leur fusil d'assaut ou leur lance-roquette hors du véhicule, sans doute pour libérer un peu de place et parvenir à s'y tenir eux-mêmes. Certains faisaient de grands signes qui émergeaient du nuage de poussière, d'autres tiraient des rafales en l'air.

Embouchure amovible ou mystérieuse composante d'une arme inconnue?

37 À ce moment-là, j'étais menotté au baraquement de police, à quelques pas de la piste et face à elle, à quelques mètres du passage des véhicules, souhaitant vivement que l'idée de prendre pour cible un nasara enchaîné ne traverse pas trop vite l'idée d'un de ces soldats sans foi ni loi.

38 Mais le temps passait, de temps à autre agité par le passage d'un pick-up vombrissant. Toujours pas de président. Je savais très bien que cela pouvait durer jusqu'au soir ainsi, et que le soleil allait chauffer. J'essayais d'entrer en contact avec un militaire qui me paraissait plus bienveillant que les autres. Mais il était moins gradé ... 
39 Finalement un véhicule blindé équipé d'une sirène annonça le passage imminent du président Déby. Nous fûmes tous contraints de nous lever, ce qui me permit bizarrement d'obtenir ma libération de l'entrave des menottes, ma demande de saluer le président en homme libre ayant curieusement abouti. Le cortège présidentiel finit par passer, constitué de limousines blanches blindées, encadrant une limousine noire au châssis allongé, parfaitement saugrenue en zone sahélienne et sur des pistes en latérite.

40 Passé le président, on laissa les paysans franchir le pont. Libre de mes mouvements, j'espérais bien alors profiter du déplacement de la foule pour me glisser jusque sur le pont et me mêler à elle. Mais un policier en civil m'en empêcha. Arrêtant une sorte de camion bâché dont le conducteur klaxonnait pour tenter de se frayer un passage, il me fit monter à ses côtés sur le plateau, non sans avoir embarqué avec lui un larron armé d'une sorte de fusil de chasse rouillé et sans doute hors d'usage. Mais fallait-il pour autant en faire la preuve et chercher à fausser compagnie à mes tristes compères ? Je choisis de n'en rien faire et de les accompagner, d'autant que le camion nous conduisait en ville et que je me sentais plus en sécurité au milieu du monde qu'en rase campagne.

41 Nous parvînmes devant une ancienne demeure coloniale décrépite, qu'on m'expliqua être le commissariat centrale de la ville. Là, on me remit entre les mains de sombres lascars qui recommencèrent à m'interroger sur les raisons de ma présence sur le passage du Président, armé d'une étrange culasse et sans le moindre papier. Ils ne semblaient pas douter que j'étais un militaire français d'un quelconque service secret et ayant comme mission d'attenter à la vie du président. J'avais beau leur expliquer que les services secrets français auraient préféré survoler le convoi avec un hélicoptère (type Tigre) pour le détruire à l'aide de quelques missiles à infrarouge (type Scalp), ils persistaient à me croire capable d'un tel exploit militaire armé d'une simple embouchure de trompette.

42 Ce n'est que le soir que le commissaire (Zaghawa) me fit libérer (son adjoint m'aurait, dit-on depuis, fait incarcérer) et reconduire à la concession de la Coopération française, muni de ma précieuse embouchure.

Épilogue en cravate

43 Nous sommes à Paris le 24/01/05, quelques semaines après mon retour du Tchad, et j'assiste à l'Assemblée nationale ${ }^{19}$ aux $12^{e}$ Rencontres parlementaires Paix et Défense, sur le thème « Nouvelles menaces : nouvelles réponses ? ", sous le haut patronage de Madame Michèle Alliot-Marie, Ministre de la défense.

44 En arrière-plan de la manifestation, le sempiternel discours que je reconnais pour l'avoir entendu maintes fois lorsque je travaillais chez Thalès ou Aérospatiale, ou lorsque j'étais Auditeur en session nationale de l'Institut des Hautes Études de Défense Nationale. En voici la substance, caractéristique du complexe politico-militaroindustriel français : «La France n'est pas aussi sauvagement opportuniste que les ÉtatsUnis d'Amérique, elle est la patrie des Droits de l'homme et du citoyen, porteuse d'un certain universalisme et d'une vision à long terme sur la paix entre les peuples; elle se bat pour un monde multipolaire et pluri-culturel; elle est un pilier de l'Europe, promeut ardemment une Défense européenne et s'investit dans le maintien de la paix et la gestion des crises à travers des accords de défense ambitieux, notamment en Afrique, comme le prouve la manière dont elle parvient à évacuer ses ressortissants dans de bonnes conditions de sécurité ... ». 
Les députés, les industriels, les militaires, la ministre de la Défense, les journalistes, tous parlent d'une seule voix, suffisante et technocratique: «Les nouvelles menaces, prolifération et terrorisme en réseau, doivent être combattues avec fermeté à l'aide de moyens guerriers plus adaptés, il faut pour cela de plus gros budgets pour développer des systèmes d'armes performants, ce qui conduira à terme à plus de paix et de justice dans le monde ». Seuls les représentants de l'Union européenne ${ }^{20}$, qui ne défendent pas de gros budgets militaires et de programmes d'équipements, osent parler des causes originaires de cette prolifération et de ses mouvements terroristes. Mais la France politico-militaro-industrielle ne daigne pas s'intéresser à des objets aussi mesquins. Quelle différence avec la position américaine ? Mis à part que celle-ci est déjà prise et qu'il convient de s'en distinguer à toute force, en quoi la position française n'est-elle pas simplement suiviste et même corollaire de la position américaine?

6 Résumons-nous : la France ${ }^{21}$ lutte contre la prolifération des armements (mais met à la mer des Sous-marins Nucléaires Lanceurs d'Engins équipés chacun de seize missiles de longue portée équipés chacun de huit têtes nucléaires de destruction massive) et lutte contre le terrorisme international (mais entraîne des Commandements des Opérations Spéciales et autres Forces spéciales ${ }^{22}$ capables d'être rapidement projetés «dans la profondeur » pour mener à bien des missions meurtrières dont aucun survivant ne pourra jamais témoigner).

À écouter ce discours, on pourrait presque croire que la France est prête à remettre le commandement de son dispositif de dissuasion au Parlement européen, à consulter les instances européennes lorsque son influence sur ses traditionnels " prés carrés » est en jeu, que les Français ne sont pas encouragés par les élus locaux et nationaux à se penser comme des Européens «supérieurs». On pourrait croire le discours que tiendrait typiquement un Ministre de la défense français si on l'interrogeait sur la coopération franco-tchadienne, et qui ressemblerait à s'y méprendre à celui de ses prédécesseurs : «La coopération franco-tchadienne est d'abord une coopération civile, dans les domaines de la santé, de l'éducation, des infrastructures et de la culture; de surcroît, à la demande des autorités tchadiennes ${ }^{23}$ la France a contracté des accords de défense ${ }^{24}$ visant au maintien de la stabilité géopolitique de la région et à l'intangibilité des frontières, dans le respect des droits de l'homme et la non-ingérence dans les affaires d'un pays souverain et ami ».

Pourtant, la réalité est tout autre ${ }^{25}$ : la France soutient Idriss Déby, forme ses troupes et sa police, comme elle l'a fait en son temps avec Tombalbaye, Félix Malloum, Hissène Habré (accusé de crimes contre l'humanité en 2000 et qui coule une paisible retraite à Dakar) et Goukouni Oueddei, sans examiner le coût humain indigène de ce soutien aveugle et intéressé : la majorité de la population est maintenue dans un état de pauvreté intense, une corruption généralisée et une répression policière et militaire sans pitié ...

A l'heure où j'écris, Idriss Déby soigne son alcoolisme à l'hôpital américain de Neuilly tandis que la population tchadienne défavorisée meurt dans des conditions atroces à cause du détournement systématique de l'aide civile et sanitaire, et ceci n'est pas étranger au fait que la France souhaite garder une base militaire permanente dans cette région de l'Afrique.

L'Afrique en miroir de l'Europe

50 Les Européens cherchent actuellement à constituer l'Europe comme monde culturel, après avoir souffert pendant plusieurs générations d'un doute profond quant à cette 
possibilitée ${ }^{26}$. Pour faire émerger une telle entité et lui laisser une place opérante dans le champ des fictions opérationnelles ${ }^{27}$ compossibles, les Européens se sont aperçus qu'il était nécessaire d'affadir une autre fiction, trop prégnante en l'état pour ne pas fonctionner comme une monade phagocytant dans l'œuf ses concurrentes: l'étatnation, forme structurante particulièrement hégémonique dans un pays comme la France.

Curieusement, l'artifice permettant à la fois d'harmoniser l'union et d'affadir les prétentions hégémoniques des l'états-nations européens dans le tumulte des fictions opérationnelles, a consisté à prendre appui sur le niveau des collectivité territoriales locales/régionales, pour pouvoir appeler l'Europe des régions en éclaireur et en renfort de l'Europe des états membres.

Remarquons que tout cela prend du temps, requiert liberté des personnes et souplesse des institutions, et mobilise une grande volonté et une énergie durable. Inutile de dire que la paix durable est nécessaire pour parvenir à ce que l'effort consenti cristallise. Or dans le même temps, que font les Européens en Afrique?

Remarquons d'abord qu'il n'existe pas encore d'Europe des affaires étrangères, et que les Européens sont redevables des seuls états membres sur ces questions, typiquement crispés sur leurs postures historiques. On est en droit de placer beaucoup d'espoirs dans une future position européenne forte, qui devra bien policer les égoïsmes nationaux des états membres et libérer les "prés carrés » de leur tutelle, comme ce sera le cas du Tchad de sa tutelle française.

Reformulons donc la question en "Que continuent à faire les états membres européens en Afrique? ». Les ressortissants des pays africains sont contraints de séjourner dans une grille interprétative de type état-nation, héritage dommageable de la colonisation, qui les défavorise, les abaisse et les inclinent aux guerres civiles pour plusieurs raisons :

Les sociétés africaines n'ont aucune affinité avec la catégorie d'état-nation, constituée au fil d'une histoire singulière par un sous-continent singulier;

Les états-sans-nation africains sont typiquement assignés à l'influence, quand ce n'est pas à la main mise, d'états-nations étrangers.

Cette situation est d'autant plus étrange que l'Afrique (limitons-nous ici à l'Afrique sahélienne) possède une forte expérience à la fois d'un niveau infra-étatique - les différentes ethnies qui peuplent la région sahélienne - et d'un niveau supra-étatique le Sahel au sens géographique, parcouru de long en large par des populations migrantes et/ou nomades.

59 L'Afrique et l'Europe pourraient donc évoluer en miroir l'une de l'autre, les deux dynamiques d'union se répondant naturellement de manière vertueuse.

En adoptant la position qu'elle maintient au Tchad, la France sacrifie une région du monde à ses intérêts supposés, l'empêchant d'affadir ses frontières d'état-sans-nation comme probablement il est nécessaire de le faire, et surtout de redessiner des entités locales plus autonomes capables de se fédérer en entités régionales trans-étatiques plus stables que les états d'aujourd'hui, qui ne recouvrent pas de réalité nationale.

61 Contraindre l'Afrique d'emprunter la voie artificielle et sinueuse dominée par la catégorie d'état-nation, alors que dans le même temps on lui interdirait l'expression de la violence afférente au long processus au nom d'un humanisme facile, est une attitude paradoxale et contre-productive. 
Pour en finir avec le cercle vicieux : mépris, terrorisme et répression

La lutte contre le terrorisme international ne doit pas rester un processus essentiellement militaire, n'en déplaise aux organisateurs des Rencontres parlementaires Paix et Défense.

Lorsque la France, dans la droite ligne des Etats-Unis d'Amérique, s'acharne dans cette posture envers ses anciennes colonies d'Afrique noire, elle génère le «terrorisme international » de demain.

64 En effet, les Africains comprennent bien de quel jeu de dupe ils font l'objet, et demain, d'une manière ou d'une autre, ils entreront en rébellion ${ }^{28}$. Les Tchadiens suivront les Ivoiriens et les Togolais, et tout le monde fera mine de s'étonner que des amis de la France se retournent «soudainement et lâchement» contre la lointaine patrie protectrice.

5 Il est urgent de prévenir ce risque en préparant au niveau européen une autre politique africaine, faisant de la construction politique et sociale de ce continent un miroir vertueux de la construction et de l'élargissement européens.

III ${ }^{\circ}$ En conclusion

En relisant ce texte, je ne peux m'empêcher d'y trouver des airs désuets de Tintin au Congo. Tintin, plus encore que Tartarin, Bonbonnel et autres chasseurs de panthères ou de fauves exotiques: on devine que Tintin est tenté de dialoguer avec les cuiseurs de briques le long du fleuve pour connaître le nom du méchant, on ne sent que trop la présence maléfique du colonel Alcazar derrière le trafic de médicaments, et on est étonné qu'un capitaine Haddock ne sorte pas de derrière les fagots pour insulter le président Déby lorsqu'il franchit le pont, et provoquer sa cohorte de moules à gaufres enturbannés et armés jusqu'aux dents. Quant aux menottes (probablement fournies par l'armée française) ayant servi à aliéner le héros aux abords du pont, elles auraient du être en ficelle de lieuse, bientôt rongées par le fidèle Milou.

67 Cette charge culturelle caricaturale ne s'invite pas par hasard. Si l'Afrique est le théâtre expérimental de nos grimaces, le laboratoire collectif de nos ambiguïtés, c'est parce que cela correspond à l'usage culturel exigé. Et cet usage est si prégnant, si bien relayé par les formes opérantes concrètes de la vie économique et sociale qu'il prescrit, que la vie en Afrique s'inscrit aujourd'hui dans cette demande extérieure, tendue et parfois déchirée entre une tradition ancestrale et des attractions en provenance d'une autre scène.

Ceci n'est possible que parce que l'Afrique, pour un occidental, est un monde radicalement autre, avec lequel aucune identification a priori n'est requise.

Esquisse 1

Michel Foucault, dans son Histoire de la folie à l'âge classique, avait bien montré que «l'enfermement des fous » coïncide en France, comme pratique institutionnelle, avec l'affirmation des dogmes fondateurs de la Révolution française. C'est seulement à partir du moment où l'autre est un alter ego, un autre moi-même, que sa folie devient infréquentable. A l'âge classique (XVII ${ }^{e}$ siècle), les fous cessent d'être omniprésents dans l'espace social pour être enfermés dans un lieu particulier, l'Hôpital général. Sont enfermés en même temps que les fous les pauvres, les mendiants, les débauchés. Ce "grand renfermement ", dit Foucault, est le corollaire du partage raison-déraison ${ }^{29}$ qui se fait explicitement en philosophie avec le «moment cartésien ». Aujourd'hui encore, l'Afrique est utilisée comme laboratoire témoin d'un âge antérieur à l'âge classique, 
même pour ceux qui ne considèrent pas les Africains comme faisant culturellement partie de l'axe de la déraison. Comme sous la Renaissance, coexistence pacifique de l'attitude critique et de l'attitude tragique, terrain d'expérience glissant et pétri de contradictions.

Freud, dans Le malaise dans la culture, avait cru voir à l'œuvre ce qu'il appelait le narcissisme de la petite différence, qui canalisait le désastre économique du monde paysan aux Etats-Unis pendant la crise de 1929 au format d'une tension sociale entre les pauvres paysans blancs et les pauvres paysans noirs. Les pauvres paysans, noirs ou blancs, ne se liguaient pas contre une bourgeoisie qui les aurait opprimés de concert en mettant à profit la mécanisation pour exacerber la concurrence : au lieu de cela, ils se jalousaient les uns les autres, reportant sur la couleur de leur peau l'injustice sociale dont ils ne se voyaient pas victimes ensemble. La tension venait s'installer au lieu de la différence la plus proche, au lieu même de la couleur de la peau. Peut-être justement parce que l'injustice sociale rendait blancs et noirs égaux devant la souffrance, fallait-il lutter avec plus de force contre sa propension égalisatrice. En Afrique, les occidentaux ne développent pas ce syndrome du narcissisme de la petite différence, ils expérimentent au contraire un monde qui ne se referme pas sur lui-même dans la sphère culturelle.

71 Ces premiers éléments d'enquête solutionnent partiellement notre interrogation de départ sur le pourquoi de l'attitude "gratuitement tolérante » qui nous habite lorsque nous fréquentons l'Afrique. Même André Gide, dans ses ouvrages Voyage au Congo et Le retour du Tchad, lorsqu'il dénonce la condition des Africains qu'il a constatée «sur le terrain » à l'occasion de son long périple, n'échappe pas complètement à cette attitude, que lui reprochent d'ailleurs avec une féroce acuité ses terribles détracteurs.

Esquisse 2

Dans La divine comédie de Dante, la figure du Christ est au centre du Jugement dernier : derrière lui, trois univers bien distincts : le Paradis, l'Enfer et le Purgatoire. Le Paradis est l'avenir recherché par les mortels, l'Enfer l'univers où le mal subi n'en finit pas de dédommager le mal commis. Théodicée élémentaire.

Par moi on va dans la cité dolente, par moi on va dans l'éternelle douleur, par moi on va parmi la gent perdue.

La divine comédie, Chant III, page 41

73 Le mortel désire sans doute être touché par la vraie vie dans cette vie-ci, pour "toute la vie durant". Mais s'il aspire à sa conversion (le "une fois pour toutes"), son espérance comme vertu théologale réside radicalement dans le motif du Paradis. Quant au savoir de la possibilité de l'Enfer, il menace le vivant dans son présent, et vise à prescrire sa conduite face au mal toujours menaçant ${ }^{30}$.

74 L'Enfer de Dante est pétri d'une rustique pédagogie divine. La gestion du mal fait retour à ce qu'elle était avant la Loi du talion, à ceci près que l'explosion de la violence a lieu ailleurs que sur Terre.

75 Car le damné sait, subit, et ne peut que gémir de remords. A l'occasion de la visite exceptionnelle d'un mortel, comme celle rendue par Dante accompagné par Virgile, il ne peut que témoigner du drame perpétuel qu'il éprouve dans sa chair torturée. Mystérieusement, l'espérance semble l'avoir définitivement quitté, conformément à l'inscription dantesque de la porte de l'Enfer.

76 La pensée du damné est tout entière regret de n'avoir pas, en son temps de vivant désormais révolu, repoussé la possibilité de commettre le mal. Le mal subi, récurrent, 
convoque éternellement les questions "Pourquoi ?" et "Pourquoi moi ?", provoquant mécaniquement la réponse réitérée "Parce que, vivant, tu as commis le péché", qui s'impose au damné.

Pour Dante, la puissance infernale est structurellement soumise à la logique. Et l'Enfer est la conséquence logique (ô combien paradoxale !) de la non-conformation à la logique.

78 Gombrowicz, dans son ouvrage Contre les poètes, va critiquer vivement cette conception. Dans l'Enfer revu par Gombrowicz, la malfaçon doit régner en abîme, et l'ordre hiérarchique laisser place à une récursivité déroutante (pages 142, puis 145-146). Et si d'aventure du café devait être servi ... il serait froid (conformément au célèbre dessin de Larson).

L'argument théorique de Gombrowicz est que le diable règne trop sérieusement dans l'Enfer de Dante pour incarner le mal métaphysique, nécessaire à la modernisation de la théodicée médiévale. Le démon dantesque fait trop bien les choses, et l'éternel cycle de souffrances suivies de regrets compulsifs, bien que conforme à notre expérience de la nature impérative de la douleur, ne lui laisse aucun rôle transcendantal.

Mais l'intuition cachée qui pousse Gombrowicz à suggérer la complexification de la structure infernale de Dante est que celle-ci, en l'état, risque de s'avérer trop plate, simple, ennuyeuse (répétitive et itérative) pour garantir la "pensée unique" des damnés.

Voyons d'abord comment Gombrowicz propose de moderniser les vers de Dante :

Par moi, l'on va dans la Cité sans fond,

éternité qui poursuit son abîme,

par moi, l'on va où le Mal éternel

s'infecte lui-même, se ronge et s'avilit,

par moi, l'on va parmi la gent perdue,

race increvable...

Sur Dante, pages 145 et 146

Et Gombrowicz de penser que la meilleure façon de prévenir le retour inopiné de l'espérance au sein même de l'Enfer est encore de déranger perpétuellement les conditions de possibilité de l'enfermement radical, en ouvrant des chausse-trappes. Le tortionnaire apprend vite (Wolfgang Sofsky, "Traité de la violence") à ajouter le vice à la torture qu'il inflige, en la donnant sur les modes imprévisibles de la cruauté. Provocation du Polonais en exil qui a fui la seconde guerre mondiale et l'occupation Soviétique ? Certes. Mais pas seulement.

83 La thèse, marquée par le structuralisme, ne semble parvenir qu'à repenser les modalités formelles de l'Enfer, sans dévoiler son essence. Mais elle prépare la possibilité théorique d'une immanence dans la transcendance : quelque chose travaille du dedans de la structure, il y a un néant agissant, un peu comme dans la grâce immanente au marxisme, ou dans un historicisme qui prétend qu'il y a de l'extériorité radicale dans l'histoire, et non pas seulement un même s'améliorant (un progrès) ou un nihilisme de la fin de l'histoire. Il peut y avoir de la subjectivité humaine mutante, entre changement de degré (le progrès du "pareil au même") et changement de nature (la conversion, avec le problème inhérent du "toute la vie").

Qu'elles sont les conditions de possibilité de cette création de subjectivité par mutation? Comment la penser ? C'était déjà la préoccupation de Nicolas de Cues au XV 
siècle lorsqu'il nous donnait sa "Docte ignorance" ou son "Guide du penseur ou du nonautre".

Michel Foucault, par exemple, prétend s'adonner à la philosophie par curiosité, mais surtout pour s'égarer de la connaissance, se déprendre de soi-même, parvenir à changer, ne plus avoir la même pensée. L'intensité non banale (qui n'est pas une simple augmentation du degré dantesque) se fait mutation en limite $: j$ 'ai à sentir quelque chose, mais je sens que pour y parvenir, je dois changer mon sentir. Il s'agit bien de "penser pour changer son penser", au contraire de l'auberge espagnole. La puissance consiste alors à nouer un renouveau subjectif autour de ces noyaux d'intensité non banale. Il n'y a pas de risque, car le risque suppose le même de celui qui risque et de celui qui fait le bilan.

Pour Gombrowicz, c'est la souffrance qui motive l'homme à penser sa pensée, contre l'indifférence :

Car la réalité est ce qui nous résiste, c'est-à-dire ce qui fait mal. Et l'homme réel est celui qui a mal (...). Supprimez la douleur et l'univers deviendra indifférent ...

Sur Dante, pages 152 et 153

Voilà donc le sens du commentaire que vient de m'adresser un ami à qui j'avais demandé de bien vouloir critiquer le présent texte :

«Mais...ce texte va très bien! D'un point de vue purement littéraire s'entend. Je crois par ailleurs deviner plutôt que déceler ce qui vous chiffonne : c'est son incohérence même, mais c'est le reflet de ... la vôtre! Ne le prenez pas mal.

Décortiquons les chose s: nous vivons dans un monde incohérent, vous avez fait un voyage incohérent, vous écrivez un texte incohérent, tout est en ordre; je trouve ça très intéressant et reflétant parfaitement l'état d'esprit de l'occidental cultivé.Vous indiquez avec justesse les contradictions de la politique française en Afrique et vous marquez en même temps les vôtres à l'occasion d'un voyage dans un pays où vous n'aviez rien à faire, où vous avez simplement fui quelques jours pour tuer le temps. Je crois que vous n'y êtes pas trop mal arrivé ...»

\section{BIBLIOGRAPHIE}

Nicolas de Cues, Du non-autre; le guide du penseur, Cerf Sagesses chrétiennes, 2002.

Alighieri Dante, La divine comédie :l'Enfer, publié à Florence en 1314, Flammarion, 1992.

Michel Foucault, L'archéologie du savoir, Gallimard, 1969.

Michel Foucault, Histoire de la folie à l'âge classique, Gallimard, 1961.

Sigmund Freud, Le malaise dans la culture, PUF Quadrige, 2002.

André Gide, Voyage au Congo suivi de Le retour du Tchad, Gallimard, 1995.

Witold Gombrowicz, Sur Dante, dans "Contre les poètes", pages 141 à 167, écrit à Vence en 1966 pour Kultura, Journal III, Ets Complexe, 1988. 
Knut Hamsun, Faim, Presses Universitaires de France, Collection Quadrige, 1994

Georges Remi Hergé, Tintin au Congo, Casterman, 1931.

Karen Kierkegaard, La reprise, Flammarion, 1990.

Leibniz, Confessio philosophi (La Profession de foi du philosophe), Vrin, 2004.

Charles de Montesquieu, Considérations sur les causes de la grandeur des Romains et de leur décadence, Garnier-Flammarion, 1968.

Jérôme Porée, Mal, souffrance, douleur, article du "Dictionnaire d'éthique et de philosophie morale", pages 904 à 911, Presses Universitaires de France, 1996.

Jean Pouillon, Le cru et le su, Seuil, 1993.

Francis Rousseaux, Le sentiment océanique, actes du groupe STP, Maison des Sciences de l'Homme, Paris, décembre 2000 (également téléchargeable à l'adresse électronique : http://

recherche.ircam.fr/equipes/sel/rousseaux/papiers/sentimentOceaniuqe.pdf)

Wolfgang Sofsky, Traité de la violence, Gallimard, 1998.

Paul Valéry, Variété I et II, Gallimard, 1998.

\section{NOTES}

1. Au Tchad, il n'est pas rare qu'une liaison routière entre deux agglomérations ne soit assurée que les jours de marché, d'où l'appellation occasion de marché des taxi-brousse dans ce pays.

2. Ces travaux ont été rassemblés en 1995 au format d'une Habilitation à Diriger des Recherches, disponible à l'adresse http://recherche.ircam.fr/equipes/sel/rousseaux/ publications.htm

3. J'avais rencontré Pierre en région parisienne à l'occasion d'un pique-nique mondain quelques années auparavant, et je l'avais invité à présenter sa récente expédition auprès des Indiens Wayampi et Émerillon de Guyane française dans le cadre d'un séminaire de sciences humaines que j'animais à Paris. Le principe d'une visite au Tchad, nouvelle affectation tout juste acceptée par Pierre, avait été convenu au café du coin après le séminaire.

4. La fin de l'année est une excellente période pour visiter le Tchad, car la saison est sèche et la chaleur acceptable, l'Harmattan n'y est pas encore trop fréquent, cette saison étant de loin la plus salubre de l'année; quelques mois plus tard, et N'Djamena devient la ville la plus chaude du monde. Ne parlons pas de la saison des pluies, que je ne connais pas mais dont j'imagine assez bien les inconvénients ...

5. On appelle nasara le blanc, qu'on ne manque jamais de remarquer, et qui fait parfois même peur aux jeunes enfants; nasara fait probablement référence au Nazaréen, par le fait que les premiers blancs venaient pour évangéliser les populations.

6. Un jour que j'avais demandé de me raccompagner à un blanc qui se trouvait être le chef de la mission ONU de déminage du Tibesti, ce citoyen suisse m'a expliqué pourquoi il s'apprêtait à jeter l'éponge : davantage de mines (antichar et antipersonnel) au Tchad qu'au Cambodge, plus de vingt systèmes techniques différents à désamorcer, des conditions de travail épouvantables (déplacement des mines avec les dunes actives, pas de pluie depuis vingt ans et donc une poussière empêchant les animaux démineurs d'intervenir - et faisant accessoirement crasher les avions de la mission, un relief 
empêchant le recours aux engins lourds de déminages), des revirements continuels du gouvernement tchadien face aux programmes d'intervention, de nombreux démineurs tués pour une surface nettoyée ridicule, après plusieurs années de mission.

7. Le cycle de confection des briques cuites dure environ cinq jours, et commence par le creusement d'une zone argileuse bien délimitée, qu'on vient détremper avec de l'eau du fleuve charriée à la brouette, jusqu'à obtenir une boue qu'on moule avec un gabarit en bois pour former les futures briques, qu'il faut laisser sécher deux jours au soleil une fois démoulées. Une fois sèches, ces briques sont ensuite soigneusement empilées en alternance avec de la paille pour constituer des pyramides enfermant du charbon de bois auquel on finit par mettre le feu pour lancer une cuisson de trois jours. Les briques cuites sont ensuite transportées à la main jusqu'aux berges du fleuve. Les briques cuites sont payées cinq francs CFA l'unité, soit environ un euro les cent vingt.

8. Il se trouve que les mêmes Zaghawas sont victimes de leurs homologues arabes dans la région du Darfour au Soudan, et que cette situation pousse les proches du président Déby à voler à leur secours, ce que l'intéressé ne se résout pas à faire. Cette tension risque fort d'écourter les jours du président, qui doit faire face à de nombreux coups d'états fomentés par des membres de son ethnie. Au Darfour, c'est donc un conflit entre blancs et noirs qui se joue, les deux camps protagonistes étant constitués de nomades islamisés.

9. Sans être expert en la matière, j'ai eu une assez mauvaise impression des ONG de petite taille, trop compromises par des intérêts très extérieurs à leurs objets prétendus : j'ai rencontré par exemple une jeune Française diplômée de Sciences Politiques, mandatée par une ONG d'une ville française jumelée avec Moundou et parachutée seule sur le terrain du Sud du Tchad pour organiser quelque spectaculaire action de prise en charge de malades atteints du SIDA. L'hôpital de Moundou étant démuni du minimum, son action a finalement consisté à accompagner jusqu'à N'Djamena un bus de malades devant subir des examens élémentaires nécessitant l'emploi d'une machine (CD4) à doser les Anti-Rétro-Viraux présents dans le sang du malade infecté par le VIH (http://www.sida-info-service.org/informer/vih/ immunitaire.php4). Dans le même temps, une autre ONG cherchait à doter l'hôpital de Moundou d'une machine à $\mathrm{CD} 4$, qui se voyait rétorquer que le projet n'était pas si urgent puisque des malades pouvaient être transportés à N'Djamena.

10. Depuis 2001, il n'existe plus de ministère de la coopération en France, mais les activités de ce type, d'ailleurs en forte restriction, sont conduites sous l'autorité directe du Ministère des affaires étrangères.

11. Pierre et moi sommes allés à la messe de minuit pour fêter Noël, dans les locaux d'une mission située non loin de la concession. À la moindre occasion, les femmes se mettaient à danser, et un vieux Père blanc prêchant en langue Ngambaye avait bien du mal à reprendre la parole après les chants. À la fin, l'église de fortune était tout entière prise par les chants et danses, les jeunes filles venaient se frotter à nous avec de grands sourires innocents, de sorte que le Christ lui-même en eut peut-être oublié (passagèrement) l'urgence de sa vocation.

12. La chose est assez courante sur les routes du Tchad, les militaires attendant des arriérés de solde dépassant souvent plusieurs mois/années de salaire, comme d'ailleurs les autres fonctionnaires; en tant qu'anciens rebelles typiquement intégrés dans l'armée régulière au fil des accords de cessez-le-feu, leur dévotion à l'institution est soumise à fluctuation, et ... Ils sont seuls à posséder des armes. 
13. Les transfusions sanguines sont même sur-pratiquées, en ce sens qu'elles sont un moyen d'extorquer de l'argent aux patients, activité à laquelle se livrent une bonne partie du personnel de l'hôpital, à commencer par le portier en passant par les sagesfemmes et sans exclure les cadres de l'hôpital eux-mêmes.

14. Ces statistiques sont obtenues à partir de données pluriannuelles issues du dépistage systématique des donneurs parents du receveur, lorsque ceux-ci sont présents et candidats au don de leur sang. Elles ne tiennent pas compte du fait aggravant que le sang des donneurs est souvent mélangé ...

15. Il est de notoriété publique que les effectifs de la seule école d'infirmiers au Tchad sont choisis par le gouvernement, et que la plupart d'entre eux ne savent pas lire, quand d'autres impétrants auraient eu le niveau requis pour réussir le concours. 16. Voir l'article "A l'embouchure jubilatoire de la trompette ", téléchargeable à l'adresse électronique http://recherche.ircam.fr/equipes/sel/rousseaux/autres2.htm 17. Pour moi qui suis trompettiste, une embouchure de trompette est une partie essentielle de l'instrument, plus métonymique encore que son pavillon ... Un peu comme la voile qu'use le poète pour évoquer le navire, j'userais volontiers de l'embouchure pour évoquer mon rapport à la trompette. Mais pour un militaire maniant les armes, une embouchure peut parfaitement passer pour métonymique d'un système d'arme.

18. On venait de me raconter quelques jours auparavant la scène suivante, qui se serait déroulée peu de temps avant à N'Djamena : un boucher réclame son dû à un militaire qui lui prend régulièrement de la viande sans jamais la payer; ce dernier, gradé de l'armée régulière, appelle un simple soldat et lui ordonne d'abattre le boucher insolent; le soldat s'exécute, et le gradé crie en direction du cadavre : alors, pourquoi ne viens-tu pas me demander ton argent? Puis les proches du boucher assassiné viennent, sans un mot, chercher le cadavre.

19. Les $12^{\grave{e}}$ Rencontres parlementaires Paix et Défense sont organisées (sic) avec le concours de Technicatome, Thalès, DCN, Eurocopter, EADS, MBDA, Dassault Aviation et Snecma. 20. Si la nouvelle constitution européenne est ratifiée, un ministre des Affaires étrangères sera nommé pour l'Union, et qui sera aussi vice-président de la Commission européenne. Le premier à inaugurer ce portefeuille sera l'actuel Monsieur PESC (Politique Européenne de Sécurité Commune), Javier Solana.

21. Je sais bien que la France est un vieil état-nation démocratique, mais cela ne constitue pas à mes yeux une catégorie d'état si clairement discriminée qu'elle devrait conférer légitimité à se doter d'équipements qui ne seraient considérés comme dangereux qu'en possession d'autres états. Sans quoi, si la démocratie servait à cela, ce serait une bien triste catégorie. Et puis, il faudrait pouvoir évacuer d'un simple revers de main l'éventualité de coups d'état, d'annexion, ou de prise de pouvoir démocratique par des tendances politiques fallacieuses.

22. http://le.cos.free.fr/index.htm

23. Suivrait un inintelligible couplet hybridant un sous-ensemble des ingrédients suivants : l'indépendance et la souveraineté des sus dites autorités, la non-ingérence dans les affaires d'un pays ami et souverain, le respect du droit international, le maintien de la stabilité géopolitique dans la région, la primauté doctrinale des solutions diplomatiques sur les solutions militaires, le respect des droits de l'homme, le développement de la démocratie, la fidélité aux engagements contractés, la noningérence dans les affaires intérieures d'un pays souverain, l'intangibilité des 
frontières, la démilitarisation des conflits, le devoir d'ingérence humanitaire, l'aide économique conditionnée à la démocratisation, l'abaissement du surendettement ... 24. http://www.cooperation.gouv.fr/mae/dcmd.html 25. Il est certain que si l'armée française quittait le Tchad du jour au lendemain, le pays plongerait rapidement dans un bain de sang; mais ce fait là ne peut pas rester seul à légitimer son maintien au long cours, car l'ensemble fait système pervers.

26. Pour revivre la situation spirituelle, culturelle et intellectuelle de l'Europe à cette époque, les Variété I et II de Paul Valéry constituent un accès irremplaçable.

27. Le terme fiction ne renvoie pas à une facticité gratuite de ce à quoi il réfère, mais bien plutôt de son institution par les hommes qui la constitue en s'y référant.

28. Voir par exemple la page Web http://www.alwihdainfo.com/articles/voir_art.php? idart $=577$

29. Une fois enfermé, le fou peut devenir objet de savoir. A la fin du XVIII siècle, la folie devient « maladie mentale ", l'asile apparaît, l'internement est médicalisé, la science psychiatrique commence à se développer. On va observer les malades mentaux, les faire écrire (d'où le nombre de textes écrits par des fous à cette époque), les traiter, souvent de manière terrible, les exhiber. C'est le triomphe de l'attitude " critique ", qui dure jusqu'à nos jours. Au XIX ${ }^{e}$ siècle et au XXe enfin, on voit ressurgir la folie sous la forme de la conscience tragique, en art (de Goya à Van Gogh), en philosophie (Nietzsche), en littérature (Artaud). Divergence maximale entre les deux attitudes qui coexistaient à la Renaissance.

30. On sait que Dante méprisait ses contemporains florentins, à qui il reprochait leur manque de profondeur et leur perpétuelle discorde. C'est pourquoi, sans doute, la vie quotidienne du damné ressemble à bien des égards à celle du dandy chez les mortels (cf. Oscar Wilde ou Guy de Maupassant), occupé à enregistrer le cours infini des transactions humaines : le nouveau, c'est "un de plus", "ça ne vaut pas mieux", "ça ne vaut pas la peine", tout revient au même dans une équivalence généralisée, appelant à la transcendance abstraite d'un lendemain peut-être meilleur sous le signe de la vraie vie ou du vrai amour, qui soustraira la négativité de la souffrance et de l'injustice. En dernier recours, la mort viendra (et le Paradis, à la grâce de Dieu), comme possibilité ultime ... A ceci près qu'en Enfer, l'étrange savoir d'une double infinité (l'éternel retour de la souffrance et la perpétuelle suspension de la mort comme fin) coupe court à toute procrastination.

\section{AUTEUR}

\section{FRANCIS ROUSSEAUX}

Francis Rousseaux est professeur des Universités, en poste à Reims (discipline "intelligence artificielle"). Il est actuellement en délégation CNRS à l'Ircam, où il coordonne des projets européens sur le thème de l'indexation sémantique de la musique et des sons. Il a été auditeur de la 50è session nationale de l'IHEDN. francis.rousseaux@univ-reims.fr 